\title{
The Drone Using an AI
}

\author{
1. V. Subapriya \\ Assistant Professor -II \\ Aarupadai Veedu Institute of Technology
}

2.Shidhin Varghese Philip
Department of CSE

Aarupadai Veedu Institute of Technology

\author{
${ }^{3}$ Noufal K \\ Department of CSE \\ Arupadai Veedu Institute of Technology
}

\author{
4. Gopinath V \\ Department of CSE \\ Arupadai Veedu Institute of Technology
}

\begin{abstract}
The drone which will be built is to help military, for agriculture, rooftop photography, and to keep an eye for illegal activities and to do more other cool activities. In this paper the drone which will be built is mainly used to track and monitor avenue crime and criminal activities which is totally done on proper time photograph technique plane which is managed and proposed via the use of particular methods the first processing will be implemented in real time two image processing techniques and second processing which unit will two take care the rest controll monitor and focused on two operation. Aircraft which is showen spherical place of five . Two kilometer which will mechanically feature that is to be operated and to be managed to operate. Detection algorithms have been implemented . The drone is most really useful accurate variety two title that shape to predefined database.
\end{abstract}

\section{INTRODUCTION}

The Computer Science technology has a high contribution for the easyness of human life. There will be many imporvements that had been made with love in the field of science and engineering field for example : Robotic Sciences, Automation etc. The merchandise and option are done through two computer systems being used in each and every subject to guide employees and two decrease the effort ot human. The increase in poverty to populace increase in one percentage in 12 months the common expansion in two 83 million crimes per year fee has been additionally increased. The crimes took most vicinity the protection can't get right of entry to swiftly such as ; road crimes and crimes below two complicated surroundings . The predominant two difficulties massive city due to the fact topics in crime which is vanished inside brief. It is very hard to trap and give up avenue two streets and crimes are too slender which will omit by the vechicle. Two vital cease the crime and to set up two peaceable massive cities via introducing a revolutionary approch .Growing wide variety now two environment friendly answer to overcome this hassle due to the fact the two human beings who are no longer concerned in crook things to do will no longer two experience free for their home and social activities.

\section{LITERATURE REVIEW}

Introduction:

I have been investigating about the topic regarding the drone for almost a month and I thought this topic is important to the field that I am studying is that the nation is much less safer even the one who has given the batch to protect us is not even caring about the people. So me as a computer science engineer student would like to build a drone that patrol's the given range of map address even in the night time by its high efficient night camera to investigate the process what is done by the strangers doing below.

\section{Existing System:}

The brief history of from the twenty century s of the world's robot in English Kirby and Service demonstrated Eigen faces method for recognition. Pent land and Turk made improvements on this research by employing Eigen faces method based on Principle Component Analysis for the same reason. PCA is a Karhumen-Loeve transformation. PCA has been ilinear dimensionality reduction method used to determine a mutual orthogonal functions which is shown in the figuare 1. It has been used the vanguard eigen vectors of the simple convariance function matrix to a lower dimension characterizetion. It has been used as a image matrix dimension reduction. For example : In a human face image will be represented in a different dimensional space like $\mathrm{g}$ dimension prompt corrective action aims to obtain an $\mathrm{h}$ dimension sub space which is used as a liear transforms which will be answered as;
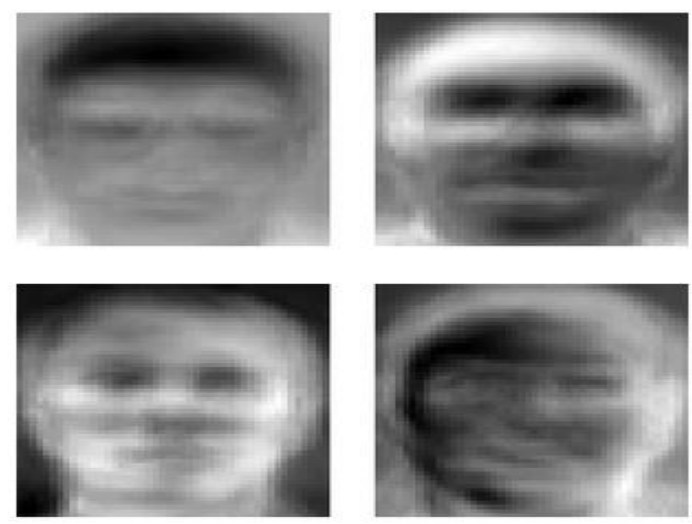

Fig 1 
A $g$ dimension space with a variance of maximum and $g$ dimension space is big due to $\mathrm{h}$. The given image can be subtracted and be normalized by using trained image by the calculated image centered images that means are calulated . If training matrix images is centered by the mean of $w$ and wi and $(b=1,2 \ldots ., 1)$ from the equation the matrix $d$ is calcualted using the trained images .covariance matrix D can be reduced down to size from the given equation. Ei and ei values are alpha which can be covariance matrix obtained . $\mathrm{Zi}=\mathrm{ET}$ wi $(\mathrm{c}=1,2, \ldots, \mathrm{d})$ is followed by the second equation , lower dimensional space is representes the new feature of $\mathrm{zi}$ to a new vector. Method that of negative aspect is scattered ias intra class to maximize the tries. Classification to intra scattering class is better for variance illuminagtion, stained classes seems very high and increase in intra class scattered

\section{$>$ Proposed System:}

We would like to build a drone for the society for the protection to the people and the world would be much safety. The much the safety the more the peacefull sleep. The drone helps the police patrol's to do the job for more secured way and the more protection to the society and the way given to the drone to look after the society safely and the whole land area given to the map by the instructor and the given way is much efficient . The drone which is built is in the safe hands like a private company or goverment so that there will no correcptions and the drone signales can be controlled by the higher autorities so the difference in the signales can be noticed by the autorities and can take a action on spot. The drone is inintiated by to catch the crime before hand so nothing bad will not happend. Drones can be damaged by the heat of sun's radiation but we are going to take that as an advantage and to charge the drone using the sun's heat so the drone will not reach zero percent if any hardware problem occures in the drone the drone have chances to fall down so we are activationg the parachute to the safe land of the drone in case of sudden hardware failure so the drone reaches the ground safely without damaging anyones property. The drone can be controlled by the google maps api so there won't be any problem to surfe through the specifc region.

\section{METHODOLOGY/ALGORITHM USED}

Approx can be reffered to the algorithm of approximation. Approx have main important 3 steps. Approx first important step is to locate the couse of an automobie tour of any goal xe $t$ to any different goal the direction and be totally different goal set but the section is of any possibel tour that can be took in FCRUP direction the constraints journey satisfies is the refueling value related in a minimu rout. The most accurate quantity is noted as the reachabel ot eh gas of a car when the car reaches a goal 1 when it should reach $\mathrm{x}$ the thinking $\mathrm{fdx}$ will also thinks in a possible way too. The automated process can go to different tour in a different vertices as a alongside the idea of the automatjion can be declared as the inovation that causes the main different details as the curriculam of the differet process as th common different things as the methodology as the alogithim is used to calculate the process as a maing differnt common things that can be declared as the main different types to be activated to solve the drone problemes that wont be solved easily and the action can be done using the given equatios as mentioned as the $\mathrm{y}$ and $\mathrm{x}$ are in a constraints with respect in the case that says can be delayesd as the attin of the shortest directin which is also direct the path of denoted through the pathe of $\mathrm{x}$ and $\mathrm{y}$ which will be the value of travelling the shortest direction in a simply fxy.

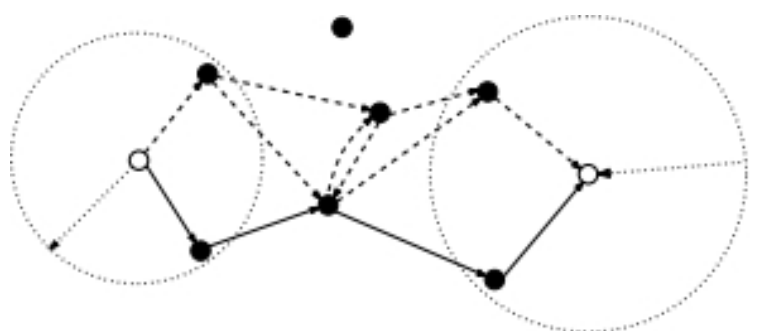

(a)Covering alogorithm with lxy that is used to cover all the target as the metric cost

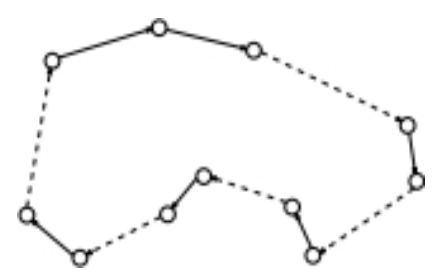

(b)coore-sponding are replaced because of the indirect edges

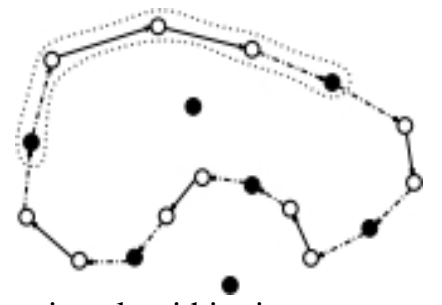

(C) appproximation algorithim just an example of the 2nd step

\section{MODULE DESCRIPTION}

\section{Photograph of the model:}

The drone which is used to take the photograph is high efficient because of the high pixel camera used in the drone. The drone is usually used to detect the crimes so we must apply the camera efficienty of the camera in the drone. The drone AI model of the camera which has been implemented in the drone is used by the government of the state or country.

\section{Videography:}

Video is usually taken from the 24 /second slide that is what video is known about and the video efficiency of the camera should be high and the camera should be a night vision. Because the drone usually fly around the area which is given to the drone using the python instructions.

\section{Evidence searching:}

The video is a $24 /$ second frame used in drone so the one by one frame which is used to take will be analysed by the given special algorithim and it will be informed to the autorites and will be took the precautions and the victim 
should be safe and the world should feel safe about the drone .

Steps in the process:

Steps involved in the given process is basically the part involved in the given process in the program of the drone and the drone is usually done by the steps of the given process of the drone's individuality of the program and the drone is self evaluated and be given to the given authorities and the process will not be much high and the drone is always secured by the self defence progress and can defend it self by the data's given by and the process is usually done by the problem and will be held and can be secured and can copy using the drone of the authorities.

\section{CONCLUSION AND FUTURE WORK}

The drone aircraft which is totally based on the function of classification extraction technique. Format of the drone aircraft is done primarily is done on two computation gadgets in picture of the processing of the operation of controll. The drone will be a great help to find the crimes on time and the culpript on time or to stop the crime or to find the crimes before that is going to take place so the drone can be an very helpful thing that can be done using the varius direcotirs of the common defaults. The drone will be secured hardively and all the signals will be encripted so the middle man can not access any of the data the drone will be trained using a very good training library so the drone can find the crimes cultivity so the program can be more cacution and if the drone has some issue the drone can handle it own so the drone will be safe the drone can be identified as the flying cop the photograph that will be take by the drone will be send to the autorities as an encrypted fiel so the midle man can't access the files will be safe. The drone assist to make two different choice in an actural time condition additionally the undertaking focus on two different classification as proposed by the mannequin the extra two environment friendly growing by the variety of the photograph is set by lowering by the processing as the better common accuray as mentioned.

\section{REFERENCES}

[1]. M.-A. Slamani and D. D. Ferris Jr, "SHAPE DETECTION," Opt. Pattern Recognit. XII, vol. 4387, pp. 176-185, 2001.

[2]. H. Pourghassem and O. Sharifi-Tehrani, and M. Nejati, "After the gun detection algorithim it took place," Aust. J. Basic Appl. Sci., vol. 5, pp. 300-307, 2011.

[3]. S. Agarwal, A. Mohan, and K. Kumar, "Desigined the construction of the UAVs," Int. J. Instrum. Control Syst. Jan2014, vol. 4, no. 1, p. 33, 2014.

[4]. H. Skinnemoen, in Electronics and Remote Sensing Technology (ICARES), 2014 IEEE International Conference on, 2014, pp. 12-19. 